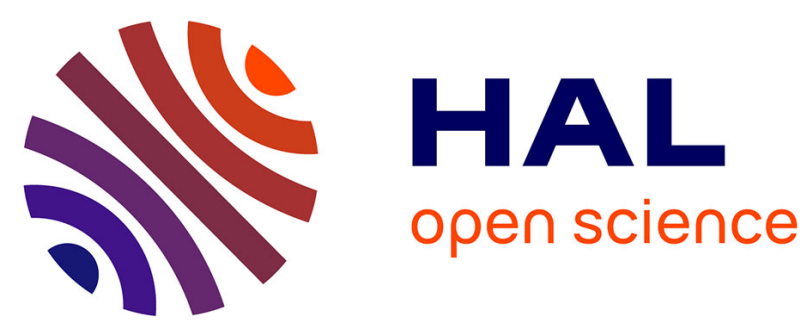

\title{
Argon surface wave discharges at medium pressure. Experiments and discussion on the energy balance
}

\author{
A. Granier, G. Gousset, P. Leprince, J. Marec
}

\section{To cite this version:}

A. Granier, G. Gousset, P. Leprince, J. Marec. Argon surface wave discharges at medium pressure. Experiments and discussion on the energy balance. Revue de Physique Appliquée, 1987, 22 (9), pp.999-1006. 10.1051/rphysap:01987002209099900 . jpa-00245660

\section{HAL Id: jpa-00245660 https://hal.science/jpa-00245660}

Submitted on 1 Jan 1987

HAL is a multi-disciplinary open access archive for the deposit and dissemination of scientific research documents, whether they are published or not. The documents may come from teaching and research institutions in France or abroad, or from public or private research centers.
L'archive ouverte pluridisciplinaire HAL, est destinée au dépôt et à la diffusion de documents scientifiques de niveau recherche, publiés ou non, émanant des établissements d'enseignement et de recherche français ou étrangers, des laboratoires publics ou privés. 


\title{
Argon surface wave discharges at medium pressure. Experiments and discussion on the energy balance
}

\author{
A. Granier, G. Gousset, P. Leprince and J. Marec \\ Laboratoire de Physique des Gaz et des Plasmas (*), Université Paris-Sud, 91405, Orsay Cedex, France
}

(Reçu le 11 février 1987, révisé le 25 mai 1987, accepté le 26 mai 1987)

\begin{abstract}
Résumé. - On étudie les décharges d'argon, créées par onde de surface à $210 \mathrm{MHz}$, dans des tubes capillaires entre 10 et 200 Torr. Ces décharges sont caractérisées expérimentalement ; le profil longitudinal de densité électronique, la fréquence de collision effective $\nu$ et la puissance moyenne nécessaire au maintien d'une paire électron-ion $\theta$ sont étudiés en fonction de la pression. On démontre expérimentalement que $\theta$ ne dépend pas de la densité électronique et est minimum entre 20 et 50 Torr. Enfin on développe un modèle énergétique simple. Il décrit bien le comportement de $\theta$ en fonction de la densité électronique et de la pression, mettant en évidence le rôle joué par la recombinaison dissociative de $\mathrm{Ar}_{2}^{+}$au-dessus de 100 Torr.
\end{abstract}

\begin{abstract}
Argon surface wave discharges, created at $210 \mathrm{MHz}$ in capillary tubes have been studied at medium pressure, from 10 Torr to 200 Torr. Such discharges have been experimentally characterized; the electron density profile along the plasma column, the effective electron neutral collision frequency $\nu$ and the mean power needed to maintain an electron-ion pair in the discharge $\theta$ have been determined as functions of the pressure. It is experimentally proven that $\theta$ is independent of the electron density and exhibits a minimum at approximately 20-50 Torr. Afterwards a simple energetic model is developed. It describes quite well the behaviour of $\theta$ with both the electron density and the pressure, indicating the dominating rôle of $\mathrm{Ar}_{2}^{+}$dissociative recombination above 100 Torr.
\end{abstract}

\section{Introduction.}

Generation and maintaining of long plasma columns by surface waves, from very low pressure $(\sim 100$ mTorr) up to atmospheric pressure, is a well known phenomenon. Up to now, these discharges have been mainly studied at low pressure ( $p \leqslant 10$ Torr), both experimentally [1-4] and theoretically [5-6].

In spite of their great importance for various applications, such as chemical analysis by optical emission spectroscopy [13-14], medium pressure discharges (10 Torr $\leqslant p \leqslant P_{\text {atm }}$ ) have seldom been investigated. Indeed in this pressure range, diagnostics and modelling are difficult to perform.

The aim of this paper is to improve the knowledge of medium pressure discharges. Therefore we have studied argon surface wave discharges in capillary tubes created at $210 \mathrm{MHz}$ from 10 Torr to 200 Torr.

First of all, we report the experimental characterization of such discharges. The axial electron density

(*) Associated with the CNRS. profile is measured and discharge characteristics, such as the effective electron neutral collision frequency for momentum transfer $\nu$ and the mean power needed to maintain an electron-ion pair in the discharge $\theta$, are determined. Next, medium pressure results are compared with those at low pressure.

Afterwards a simple energetic model, yielding approximate values of $\theta$ is exhibited. Finally the influence of both electron density and pressure on the power required to maintain the discharge is discussed, and the results of the model are compared with those of the experiment.

\section{Experimental characterization of the medium pressure discharges.}

2.1 THE EXPERIMENTAL SET-UP. - The experimental set-up is shown in figure 1. The discharge is created in a capillary quartz tube (internal radius, $a$, ranges from 1 to $1.75 \mathrm{~mm}$ ) in argon gas, without flow. The HF power (up to $400 \mathrm{~W}$ ) is coupled to the argon gas by a surfatron structure [1]. With such a 


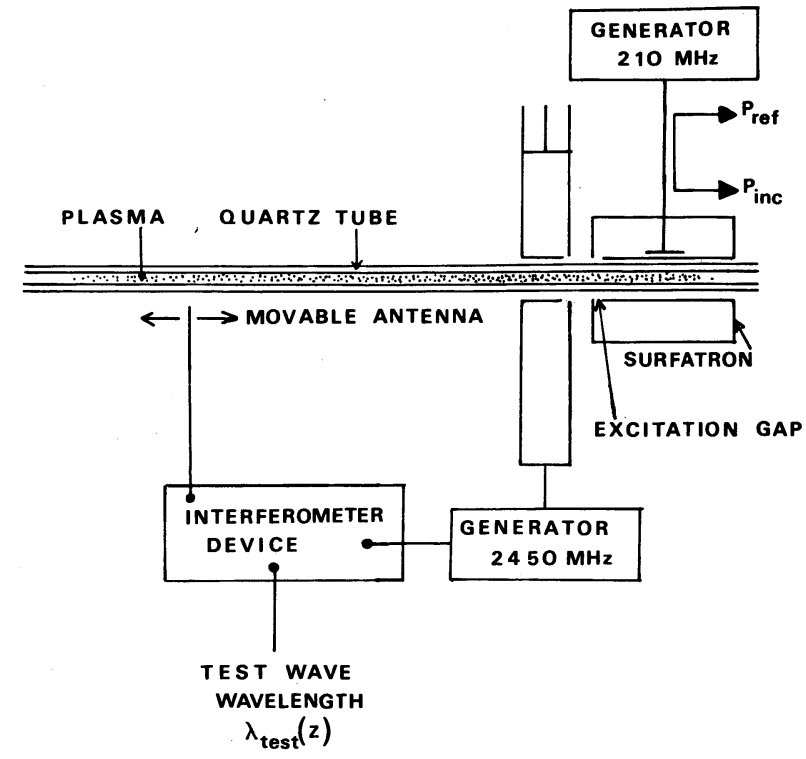

Fig. 1. - The experimental set-up.

launcher, the symmetric azimuthal mode $m=0$ is preferentially excited. Incident $\left(P_{\text {inc }}\right)$ and reflected $\left(P_{\text {ref }}\right)$ powers are measured by a bidirectional coupler. Coupling efficiency $\left(1-P_{\text {ref }} / P_{\text {inc }}\right)$ depends on the pressure and ranges from $60 \%$ (200 Torr) to $95 \%$ (10 Torr).

The surface wave simultaneously propagates and ionizes from the excitation gap to the end of the plasma column. The power carried by the wave, as well as the electron density decrease from the excitation gap to the end of the discharge, where the electron density is equal to a critical value $n_{\mathrm{e}}^{\mathrm{c}}$, which is an increasing function of the wave frequency.

The abscissa $Z=0$ is chosen at the end of the discharge and the excitation gap abscissa is $Z=L$, where $L$ denotes the plasma length.

2.2 Axial Electron Density PROFIle. - In medium pressure discharges, the electron density ranges from a few $10^{12} \mathrm{~cm}^{-3}(Z=0)$, to a few $10^{14} \mathrm{~cm}^{-3}(Z=L)$. The electron density is difficult to measure over such a range. Thus, the diagnostic generally used in low pressure and low collisional discharges has been modified to enable the electron density diagnostic under our collisional plasma conditions.

Indeed, in low pressure surface wave discharges the effective collision frequency $\nu$ is smaller than the excitation pulsation $\omega$. Thus the dispersion relation of the exciting wave $\lambda\left(n_{\mathrm{e}}\right)$ is independent of $\nu$. Furthermore, the axial wavelength profile of the exciting wave $\lambda(Z)$ can easily be measured by an interferometer method, which has been accurately detailed previously $[4,7]$. Then, the axial electron density profile $n_{\mathrm{e}}(Z)$ is deduced from the dispersion relation $\lambda\left(n_{\mathrm{e}}\right)$. Nevertheless, in medium pressure discharges created at $210 \mathrm{MHz}$, the electron-neutral collision frequency is much greater than the excitation pulsation (i.e. $\nu \gg \omega$ ). Then the dispersion curve $\lambda\left(n_{\mathrm{e}}\right)$ strongly depends on $\nu$ [8], which makes it difficult to determine the electron density from the wavelength of the exciting wave.

Therefore a low power test wave is excited at a frequency $f_{\text {test }}$ in the discharge created at the frequency $f_{0}[8,9]$. We have used a test frequency $(2450 \mathrm{MHz})$ greater than the excitation frequency $(210 \mathrm{MHz})$ so that the ratio $\nu / \omega_{\text {test }}$ is about 1 . Furthermore, since the critical density of the test wave is greater than the critical density of the exciting wave, the test wave stops propagating before the end of the discharge, avoiding the development of any stationary wave.

This enables the determination of the electron density from the wavelength profile of the test wave, without taking into account $\nu / \omega\left(0 \leqslant \nu / \omega_{\text {test }} \leqslant 2\right)$, since in our electron density range all the corresponding curves $\lambda\left(n_{\mathrm{e}}\right)$ coincide. The axial electron density profile obtained at 100 Torr $(a=1 \mathrm{~mm})$ is plotted in figure 2.

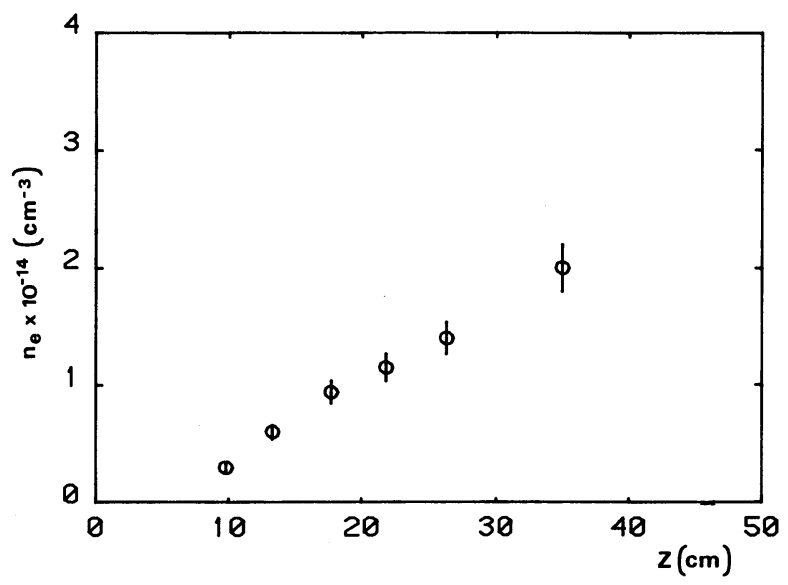

Fig. 2. - A typical electron density profile. $p=100$ Torr, $a=1 \mathrm{~mm}, P_{\text {inc }}=250 \mathrm{~W}, L=50 \mathrm{~cm}$.

2.3 MEAN POWER NEEDED TO MAINTAIN AN ELECTRON. - At a given pressure, $n_{\mathrm{e}}(Z)$ has been measured for various incident powers. Taking the origin at the end of each plasma column, we note that the different axial profiles $n_{\mathrm{e}}(Z)$ are fitting. Thus we can unambiguously define in every plasma slab (defined by its abscissa $Z$ and its thickness $\Delta Z$ ), the mean power needed to maintain an electron in the discharge $\theta(Z)$. Thus $\theta(Z)$ is equal to :

$$
\theta(Z)=P_{\text {abs }}(Z, \Delta Z) / N_{\mathrm{e}}(Z, \Delta Z)
$$

$P_{\text {abs }}(Z, \Delta Z)$ is the absorbed power in the considered slab,

$$
P_{\mathrm{abs}}(Z, \Delta Z)=P_{\mathrm{inc}}(Z+\Delta Z / 2)-P_{\mathrm{inc}}(Z-\Delta Z / 2)
$$


$N_{\mathrm{e}}(Z, \Delta Z)$ is the total number of electrons which are contained in this slab

$$
N_{\mathrm{e}}(Z)=\Pi a^{2} \cdot \int_{Z-\Delta Z / 2}^{Z+\Delta Z / 2} n_{\mathrm{e}}(\eta) \mathrm{d} \eta .
$$

Typical results of $\theta(Z)$ are plotted in figure 3 $(p=100$ Torr, $a=1 \mathrm{~mm}$, and $\Delta Z=10 \mathrm{~cm}$ ). $\theta$ accuracy is about $20 \%$. Thus we deduce that $\theta$ is almost constant along the discharge, i.e., $\theta$ does not depend on the electron density. We conclude that $\theta$ can be considered as depending only on the pressure and on the discharge radius. Thus $\theta$ is a characteristic of the discharge. This property has previously been pointed out in low pressure discharges $[4,6]$ but was not expected in medium pressure discharges. The aim of next sections is to explain and discuss this experimental result, using an energy balance in every plasma slab.

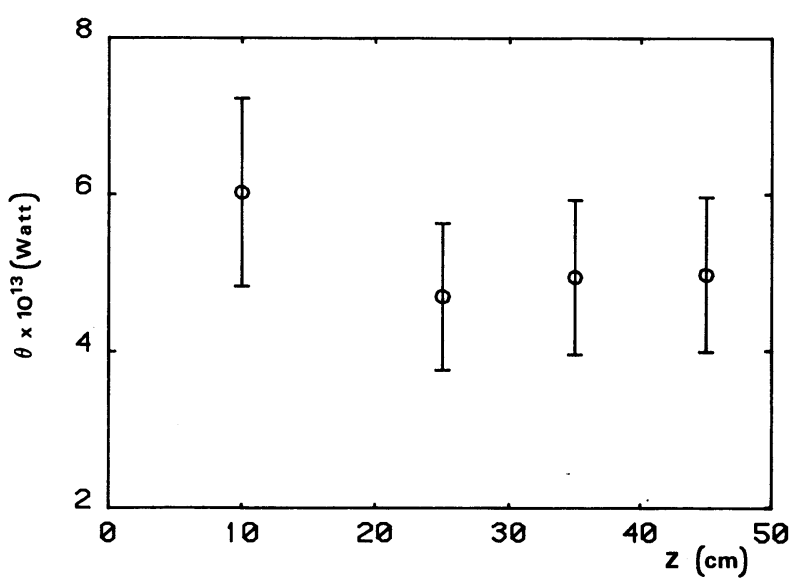

Fig. 3. - A typical mean power for maintaining the discharge profile. $p=100$ Torr, $a=1 \mathrm{~mm}, P_{\mathrm{inc}}=250 \mathrm{~W}$, $L=50 \mathrm{~cm}$.

2.4 EFFECTIVE COLLISION FREQUENCY. - Until now we have measured the axial profile $n_{\mathrm{e}}(Z)$ and defined a power $\theta$, characteristic of the discharge. If we further assume that the effective collision frequency $\nu$ is constant along the discharge, we can easily compute axial electron density profiles $n_{\mathrm{e}_{\nu}}^{\text {th }}(Z)$ depending on $\nu$. Indeed, we can write in any slab $(Z$, $\mathrm{d} Z$ ) that the power lost by the wave equals the power needed to maintain the plasma,

$$
P_{\text {inc }}(Z) \cdot 2 \alpha_{\nu}\left(n_{\mathrm{e}}(Z)\right) \cdot \mathrm{d} Z=\theta \cdot \Pi a^{2} \cdot n_{\mathrm{e}}(Z) \cdot \mathrm{d} Z
$$

$\alpha$ is the attenuation of the surface wave. $\alpha$ is deduced from the wave propagation study [8] and depends on $a, \omega, \nu$ and $n_{\mathrm{e}}$. Since $a$ and $\omega$ are fixed (for a given discharge), $\alpha$ can be written in the form $\alpha_{\nu}\left(n_{\mathrm{e}}\right)$.

Differentiation of relation (2), taking into account that $\mathrm{d} \theta / \mathrm{d} Z=0$ and assuming $\nu$ independent of $Z$, leads to the following relation [8],

$$
\frac{\mathrm{d} n_{\mathrm{e}}}{\mathrm{d} Z}=\frac{2 \cdot \alpha_{\nu}\left(n_{\mathrm{e}}\right) \cdot n_{\mathrm{e}}}{1-\frac{\mathrm{d} \alpha_{\nu}}{\mathrm{d} n_{\mathrm{e}}} \cdot \frac{n_{\mathrm{e}}}{\alpha_{\nu}}}
$$

which is numerically integrated to provide theoretical electron density profiles $n_{\mathrm{e}_{\nu}}^{\text {th }}(Z)$.

Then we can compare the experimental electron density profile $n_{\mathrm{e}}^{\exp }(Z)$ with theoretical profiles, computed for various values of $\nu, n_{\mathrm{e}_{\nu}}^{\text {th }}(Z)$. The best fit of $n_{\mathrm{e}_{\nu}}^{\text {th }}(Z)$ with $n_{\mathrm{e}}^{\text {exp }}(Z)$ leads to the effective collision frequency $\nu$. An example of $\nu$ determination is exhibited in figure $4(p=100$ Torr, $a=1 \mathrm{~mm}$, $\nu=2.6 \times 10^{10} \mathrm{~s}^{-1}$ ). From this example we deduce that $\nu$ is obtained with about $20 \%$ accuracy.

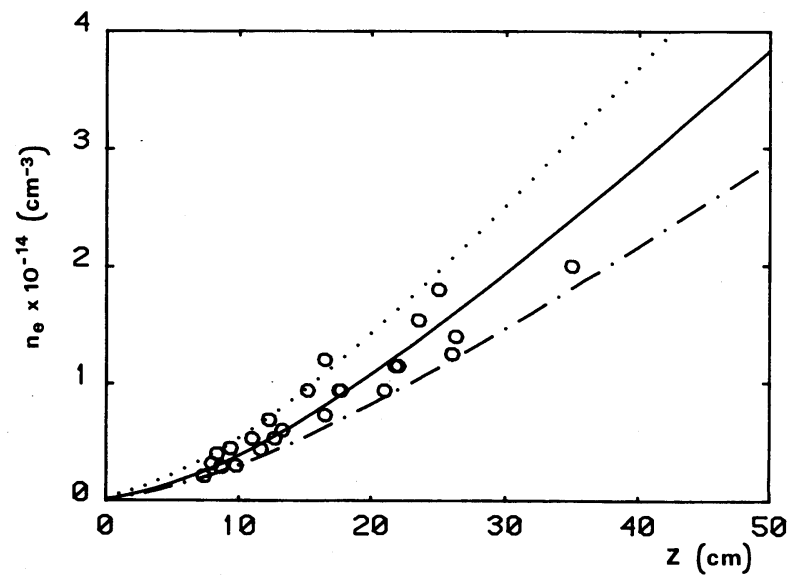

Fig. 4. - Determination of the effective collision frequency, $p=100$ Torr, $a=1 \mathrm{~mm}, P_{\text {inc }}=250 \mathrm{~W}, L=50 \mathrm{~cm}$. (O) experimental electron density. Calculated electron density profile for various collision frequencies $\left(\square \cdot-{ }^{-}\right) \quad \nu=1.95 \times 10^{10} \mathrm{~s}^{-1}, \quad(\square)$ $\nu=2.6 \times 10^{10} \mathrm{~s}^{-1},(\ldots.) \nu=3.25 \times 10^{10} \mathrm{~s}^{-1}$.

\section{Experimental results versus pressure.}

In the previous section we have developed a method to experimentally characterize a discharge. We are now going to study the variation of the discharge characteristics with the pressure. $\nu$ and $\theta$ variations are plotted in figures 5 and 6 . These results have been obtained at medium pressure (10 Torr-200 Torr) in two different tubes $(a=1 \mathrm{~mm}$ and $a=1.75 \mathrm{~mm}$ ) and low pressure results [7] have been plotted too.

Such experiments have not been performed above 200 Torr because of the constriction of the discharge (which is experimentally observed). Indeed such a constriction disables the diagnostic of $\nu$ and $\theta$. Therefore the incertainty of $\nu$ and $\theta$, is greater at 200 Torr (constriction limit) than at lower pressures. 


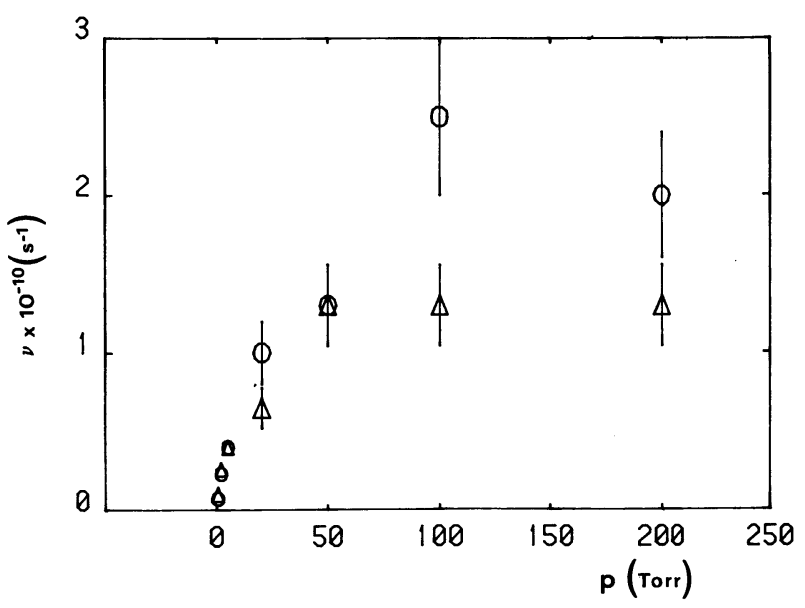

Fig. 5. - Dependence on the pressure of the collision frequency in several tubes. Values of $a(\mathrm{~mm}),(0), 1 ;(\Delta)$, 1.75. Large symbols are used for medium pressure results.

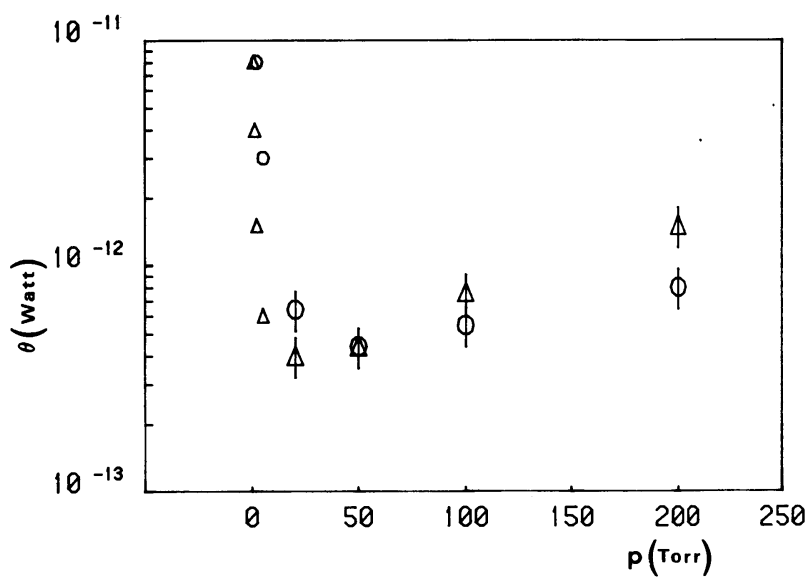

Fig. 6. - Dependence on the pressure of the mean power $\boldsymbol{\theta}$ for maintaining discharges in several tubes. Values of $a$ $(\mathrm{mm})$ : $(\mathrm{O}), 1 ;(\triangle), 1.75$. Large symbols are used for medium pressure results.

First of all we note that medium pressure results are in fair agreement with low pressure ones. No discontinuity is observed. The effective collision frequency increases with the pressure ; at low pressure $\nu$ is nearly proportional to the pressure while at high pressure $\nu$ increases only slightly with the pressure.

Moreover, the ratio $\nu / \omega_{\text {test }}$ is smaller than 2 for all pressures. This validates the use of the test wave dispersion curve $\lambda\left(n_{\mathrm{e}}\right)$ independent of $\nu$ (cf. Sect. 2).

Nevertheless, measurements of $\theta$ show a marked difference between low and medium pressures. Indeed, at low pressure, $\theta$ strongly decreases as the pressure increases. Then $\theta$ reaches a minimum, before increasing with the pressure at higher pressures. The pressure corresponding to the minimum of $\theta$ depends on the plasma radius. $\theta$ is minimum at : $\sim 20$ Torr with $a=1.75 \mathrm{~mm}$

$\sim 50$ Torr with $a=1 \mathrm{~mm}$.

Since $\theta$ is the power needed to maintain an electron in the discharge, it obviously depends on the electron loss processes, and a minimum of $\theta$ corresponds to a minimum of electron losses.

The study of low pressure discharges has proven that such discharges are governed by ambipolar diffusion [5-6], so that electrons are mainly lost by ambipolar diffusion and recombination on the tube walls. The ambipolar diffusion electron loss frequency decreases as the pressure increases, which explains that $\theta$ has the same behaviour at low pressure.

The increase of $\theta$ at higher pressures means that another electron loss process occurs which increases the electron loss frequency. We expect this loss process to be volume recombination of $\mathrm{Ar}^{+}$or $\mathrm{Ar}_{2}^{+}$.

So the minimum of $\theta$ corresponds to a change in the plasma behaviour and occurs when ambipolar diffusion and volume recombination electron losses are comparable.

\section{Simple energetic model.}

In the previous section we have qualitatively discussed the variations of $\theta$ with the pressure. The aim of this section is to make an approximate calculation of $\theta$, enabling us to understand its variations with both the electron density and the pressure.

$\theta$ is the energy transferred per electron (which takes its energy from the microwave electric field) and per second to the surrounding argon gas. This power is transferred either by elastic collisions or by inelastic collisions (excitation and ionization). Our aim is to calculate an approximate value of $\theta$ versus $n_{\mathrm{e}}, p, a$, under our experimental conditions. Therefore we shall use a three step method. Firstly we write an exact expression of $\theta$. Next, we shall discuss how to approximate this expression. Finally we shall develop a simple kinetic model, enabling us to obtain approximate values of $\theta$.

4.1 EXACT EXPRESSION OF $\boldsymbol{\theta}$. - From its definition, $\theta$ can be written as the sum of three terms, $\theta_{\mathrm{el}}$ the power dissipated by electron-neutral elastic collisions, $\theta_{\text {ion }}$ the power transferred by ionization, either by direct or multistep ionization and $\theta_{\text {exc }}$ the power transferred by excitation to any upper level, without contribution to the ionization.

$$
\theta=\theta_{\mathrm{el}}+\theta_{\text {ion }}+\theta_{\mathrm{exc}} .
$$

Using the following notations, we can write detailed expressions of $\theta_{\mathrm{el}}, \theta_{\mathrm{ion}}$, and $\boldsymbol{\theta}_{\mathrm{exc}}$.

$m$ : electron mass 
$M$ : argon mass

$T_{\mathrm{e}}$ : electron temperature

$\nu$ : electron-neutral collision frequency

$V_{j}$ : threshold excitation energy of a particular atomic state $j$

$V^{+}$: argon ionization potential

$n_{j}: j^{\text {th }}$ excited level density

$C$ : electron rate coefficient with a subscript and an upper subscript denoting respectively the initial and the final states of a transition, (subscript $j=0$ refers to the ground state and upper subscript + means ionization)

$\nu_{j}^{+}$: ionization frequency per electron $\left(=n_{j} C_{j}^{+}\right)$

$\nu_{q}^{m}$ : excitation frequency per electron $\left(=n_{q} C_{q}^{m}\right)$

- $\theta_{\mathrm{el}}$ can be written as

$$
\theta_{\mathrm{el}}=\frac{3 m}{M} k T_{\mathrm{e}} \nu
$$

where $k$ is the Boltzmann constant

- Whatever the ionization process (direct or multistep) the energy transferred by ionization is equal to $\mathrm{eV}^{+}$. In a multistep ionization the energy is transferred by electron collisions to an excited level $k$ followed by an ionization from this excited level, which frequency is denoted $\nu_{k}^{+}$. Finally the total power absorbed by ionization can be written in the form :

$$
\theta_{\text {ion }}=e V^{+} \sum_{k=0}^{\infty} \nu_{k}^{+}
$$

Thus we introduce an effective ionization collision frequency

$$
\nu_{\text {ion }}^{\text {eff }}=\sum_{k=0}^{\infty} \nu_{k}^{+}
$$

and relation (6) becomes

$$
\theta_{\text {ion }}=e V^{+} \nu_{\text {ion }}^{\text {eff }}
$$

- $\theta_{\text {exc }}$ is equal to the total energy transferred from any excited level to any upper level minus the power involved in a multistep ionization process,

$$
\theta_{\text {exc }}=\sum_{q=0}^{\infty} \sum_{\substack{m=1 \\ m>q}}^{\infty} e\left(V_{m}-V_{q}\right) \nu_{q}^{m}-\sum_{k=1}^{\infty} e V_{k} \nu_{k}^{+}
$$

4.2 Estimation of $\theta$. - The expression of $\theta$ involves the excitation and ionization cross sections (involved in the various rate coefficients $C$ ) as well as the populations of the various excited levels and the electron distribution function. So the exact calculation of $\theta$ requires, on the one hand, the knowledge of all the cross sections and, on the other hand the resolution of a complete collisional radiative model. This model consists in the resolution of the steady state rate balance equation for electrons, ions, and both excited or ground state atoms. It has been solved at low pressures $[5,6]$ but not at medium pressure. Indeed at higher pressure many more species (particularly $\mathrm{Ar}_{2}^{+}$) and many more processes (particularly three body reactions) have to be taken into account. Moreover many reaction rate coefficients are unknown. Our aim is not to solve such a complex system, but to find approximate values of $\theta$.

First of all, $\theta_{\mathrm{el}}$ is relatively easy to estimate. Indeed $\nu$ is determined experimentally and $T_{\mathrm{e}}$ is about $10^{4} \mathrm{~K} \cdot \boldsymbol{\theta}_{\mathrm{el}}$ ranges from $10^{-14} \mathrm{~W}$ (at 1 Torr) to $10^{-13} \mathrm{~W}$ (at 200 Torr).

Secondly, $\theta_{\text {ion }}=e V^{+} \nu_{\text {ion }}^{\text {eff }}$ cannot be easily calculated since it would require a complete collisional radiative model. Nevertheless, since creations and losses of electrons are equal, the effective ionization frequency $\nu_{\text {ion }}^{\text {eff }}$ per electron is equal to the effective electron loss frequency $\nu_{\text {loss }}^{\text {eff }}$ per electron

$$
\nu_{\text {ion }}^{\text {eff }}=\nu_{\text {loss }}^{\text {eff }}
$$

and

$$
\theta_{\text {ion }}=e V^{+} \nu_{\text {loss }}^{\text {eff }}
$$

So we shall calculate $\nu_{\text {loss }}^{\text {eff }}$ instead of $\nu_{\text {ion }}^{\text {eff }}$, since it involves rather well-known processes (i.e. diffusion and volume recombination).

On the other hand $\theta_{\text {exc }}$ cannot be estimated, since it can only be written in the form (9), implying the resolution of a complete collisional radiative model.

In conclusion, our aim is now to estimate $\boldsymbol{\theta}_{\text {ion, }}$ which will enable us to compare the experimental values of $\theta$ with the estimated values $\boldsymbol{\theta}_{\mathrm{el}}+\boldsymbol{\theta}_{\text {ion }}$

\subsection{ESTIMATION OF $\theta_{\text {ion }}$.}

4.3.1 Determination of the electron loss frequency. - We shall estimate the effective loss frequency taking into account the various loss processes. This study is achieved under the following conditions :

pressure (Torr) $\quad: 1$ Torr $\leqslant p \leqslant 200$ Torr electron density $\left(\mathrm{cm}^{-3}\right): 10^{12} \mathrm{~cm}^{-3} \leqslant n_{\mathrm{e}} \leqslant 10^{15} \mathrm{~cm}^{-3}$ electron temperature $(\mathrm{K}): T_{\mathrm{e}} \simeq 10^{4} \mathrm{~K}$ neutral temperature (K) : $1000 \mathrm{~K} \leqslant T_{0} \leqslant 2000 \mathrm{~K}$ plasma radius $(\mathrm{cm}) \quad: 0.1 \mathrm{~cm} \leqslant a \leqslant 0.175 \mathrm{~cm}$

The units used in any numerical expression are given inside the brackets.

Charged particles are lost, either by ambipolar diffusion followed by recombination on the wall, or by volume recombination. Thus $\nu_{\text {loss }}^{\text {eff }}$ can be written as the sum of two loss frequencies $\nu_{\mathrm{A}}$ (ambipolar diffusion) and $\nu_{R}$ (volume recombination)

$$
\nu_{\text {loss }}^{\text {eff }}=\nu_{\mathrm{A}}+\nu_{\mathrm{R}} \text {. }
$$


The ambipolar diffusion loss frequency can be written in the form (assuming a Bessel radial profile)

$$
\nu_{\mathrm{A}}=\left(\frac{2.4}{a}\right)^{2} D_{+}\left(1+T_{\mathrm{e}} / T_{+}\right) .
$$

$D_{+}$is the ambipolar diffusion coefficient which is nearly the same for $\mathrm{Ar}^{+}$and $\mathrm{Ar}_{2}^{+}$ions. $T_{+}$is the ion temperature which is assumed to be equal to the neutral gas temperature (indeed the ions are weakly influenced by the electric field). Finally $\nu_{\mathrm{A}}$ is equal to

$$
\nu_{\mathrm{A}}=2.2 \times 10^{-3} T_{0}\left(T_{0}+T_{\mathrm{e}}\right) / p a^{2}
$$

or

$$
\nu_{\mathrm{A}}=2.15 \times 10^{16} T_{\mathrm{e}}\left(1+T_{0} / T_{\mathrm{e}}\right) / n_{0} a^{2}
$$

$\nu_{\mathrm{A}}$ mainly depends on the neutral density $n_{0}$, the electron temperature and the plasma radius. Under our conditions $\nu_{\mathrm{A}}$ ranges from $10^{4} \mathrm{~s}^{-1}$ (200 Torr) to $2 \times 10^{6} \mathrm{~s}^{-1}$ (1 Torr).

To express $\nu_{R}$, both the three-body collisional radiative recombination of $\mathrm{Ar}^{+}$(frequency $\nu_{\mathrm{R}}^{\mathrm{CR}}$ ) and the dissociative recombination of $\mathrm{Ar}_{2}^{+}$(frequency $\nu_{\mathrm{R}}^{\mathrm{D}}$ ) have to be taken into account.

$$
\begin{aligned}
& \nu_{\mathrm{R}}=\nu_{\mathrm{R}}^{\mathrm{CR}}+\nu_{\mathrm{R}}^{\mathrm{D}} \\
& \nu_{\mathrm{R}}^{\mathrm{CR}}=\alpha_{\mathrm{CR}}\left[\mathrm{Ar}^{+}\right] \\
& \nu_{\mathrm{R}}^{\mathrm{D}}=\alpha_{\mathrm{D}}\left[\mathrm{Ar}_{2}^{+}\right]
\end{aligned}
$$

$\alpha$ denotes the recombination coefficient and is expressed in $\mathrm{cm}^{3} \mathrm{~s}^{-1} \cdot \alpha_{\mathrm{CR}}$ depends on electron density and electron temperature and has been calculated from Chen's values [10]. Under our conditions $\alpha_{\mathrm{CR}}$ ranges from $10^{-12} \mathrm{~cm}^{3} \mathrm{~s}^{-1}$ to $10^{-11} \mathrm{~cm}^{3} \mathrm{~s}^{-1}$. On the other hand $\alpha_{D}$ only depends on electron and neutral temperatures. $\alpha_{D}$ has been calculated from T.F O'Malley et al. values [11] and is about $10^{-8} \mathrm{~cm}^{3} \mathrm{~s}^{-1}$.

Nevertheless the main recombination loss process can only be determined if $\mathrm{Ar}_{2}^{+}$and $\mathrm{Ar}^{+}$densities are known. First of all, $\left[\mathrm{Ar}^{+}\right]$and $\left[\mathrm{Ar}_{2}^{+}\right]$are related to $n_{\mathrm{e}}$ by the neutrality condition,

$$
\left[\mathrm{Ar}^{+}\right]+\left[\mathrm{Ar}_{2}^{+}\right]=n_{\mathrm{e}} \text {. }
$$

Secondly $\left[\mathrm{Ar}_{2}^{+}\right]$is deduced from its steady state equation, assuming $\mathrm{Ar}_{2}^{+}$is created by three-body collisions $\left(\mathrm{Ar}^{+}+2 \mathrm{Ar} \rightarrow \mathrm{Ar}_{2}^{+}+\mathrm{Ar}\right)$ which rate constant is denoted $K$ and equals $2.2 \times 10^{-31} \mathrm{~cm}^{6} \mathrm{~s}^{-1}$ [12] and destroyed by dissociative recombination

$$
\frac{\partial\left[\mathrm{Ar}_{2}^{+}\right]}{\partial \mathrm{t}}=K n_{0}^{2}\left[\mathrm{Ar}^{+}\right]-\alpha_{\mathrm{D}} n_{\mathrm{e}}\left[\mathrm{Ar}_{2}^{+}\right]=0 \text {. }
$$

Equations (19) and (20) yield $\mathrm{Ar}^{+}$and $\mathrm{Ar}_{2}^{+}$ densities as functions of neutral and electron densities and temperatures. Thus they yield the recombination loss frequencies $\nu_{\mathrm{R}}^{\mathrm{CR}}$ and $\nu_{\mathrm{R}}^{\mathrm{D}}$ [(17) and (18)].
4.3.2 Variation of $\nu_{\text {loss }}^{\text {eff }}$ with the pressure and the electron density. - The effective loss frequency is the sum of three terms

$$
\nu_{\text {loss }}^{\text {eff }}=\nu_{\mathrm{A}}+\nu_{\mathrm{R}}^{\mathrm{CR}}+\nu_{\mathrm{R}}^{\mathrm{D}} \text {. }
$$

We have plotted the variations of $\nu_{A}, \nu_{R}^{C R}$, $\nu_{\mathrm{R}}^{\mathrm{D}}$, versus the pressure for various electron densities $\left(n_{\mathrm{e}}=5 \times 10^{12}, 5 \times 10^{13}, 5 \times 10^{14} \mathrm{~cm}^{-3}\right)$ in figure 7 .

First of all we note that, whatever the pressure and the electron density, $\nu_{R}^{C R}$ is negligible with regards to $\nu_{\mathrm{R}}^{\mathrm{D}}$. We conclude that the dominant recombination process is dissociative recombination of $\mathrm{Ar}_{2}^{+}$, which means that $\mathrm{Ar}_{2}^{+}$plays a dominant role in the discharge.

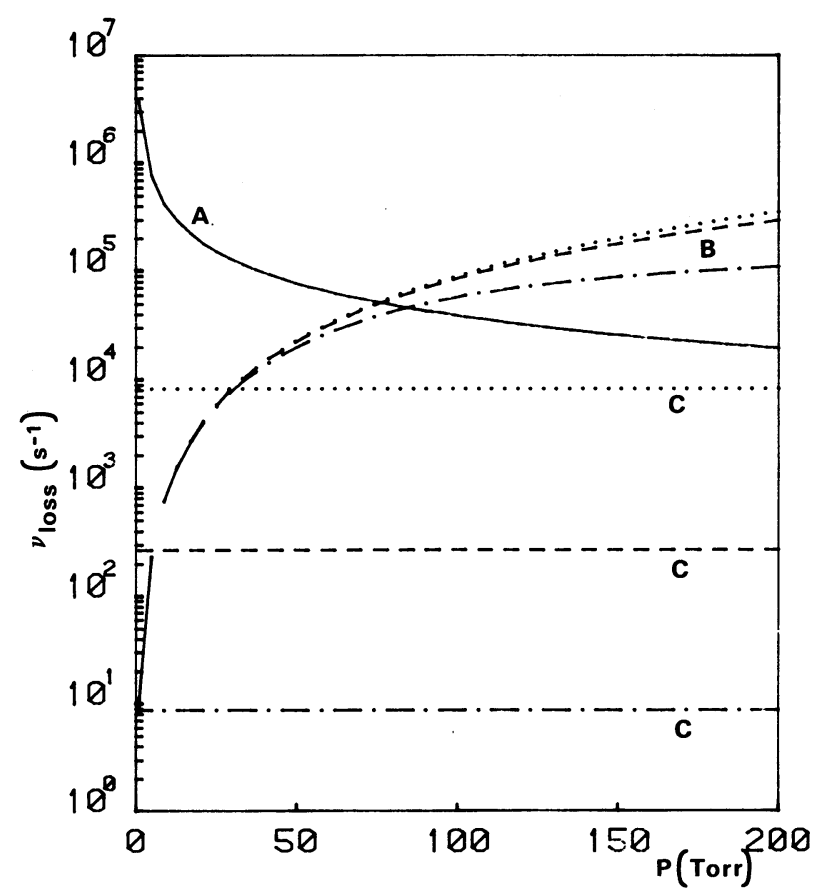

Fig. 7. - Various loss frequencies versus the pressure assuming $T_{\mathrm{e}}=10^{4} \mathrm{~K}, T_{0}=1500 \mathrm{~K}, a=1 \mathrm{~mm}$. Various loss processes: (A) ambipolar diffusion, (B) dissociative recombination (C) collisional-radiative. Values of $n_{\mathrm{e}}$ : $(-\cdot-) 5 \times 10^{12} \mathrm{~cm}^{-3},(--) 5 \times 10^{13} \mathrm{~cm}^{-3}, \quad(\ldots)$ $5 \times 10^{14} \mathrm{~cm}^{-3}$.

Secondly, figure 7 illustrates the dominant electron loss processes as a function of pressure. The discharge is governed by ambipolar diffusion up to about 10 Torr while dissociative recombination dominates above 100 Torr. Since $\nu_{\mathrm{A}}$ decreases as $p$ increases and $\nu_{\mathrm{R}}$ increases with $p$, the effective loss frequency exhibits a minimum when ambipolar diffusion and dissociative recombination are comparable.

Lastly, we consider the variations of $\nu_{R}=\nu_{R}^{\mathrm{D}}$ with electron density. Both the $\mathrm{Ar}_{2}^{+}$density $\left[\mathrm{Ar}_{2}^{+}\right]$and 


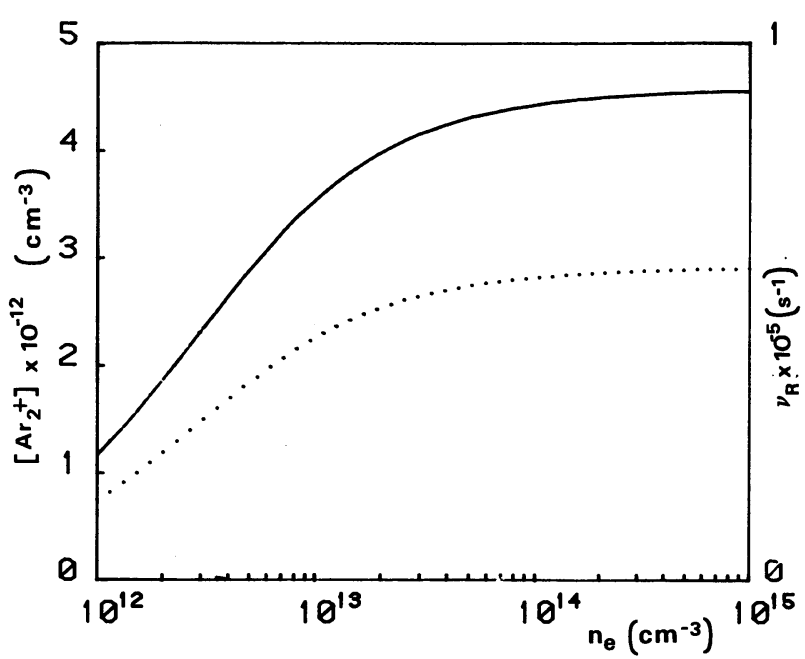

Fig. 8. - Dependence on the electron density of the estimated dissociative recombination loss frequency (—) and $\mathrm{Ar}_{2}^{+}$density (..), assuming $a=1 \mathrm{~mm}$, $T_{\mathrm{e}}=10^{4} \mathrm{~K}, \quad T_{0}=1500 \mathrm{~K}, \quad p=100$ Torr $\quad$ (i.e., $\left.n_{0}=6.5 \times 10^{17} \mathrm{~cm}^{-3}\right)$.

$\nu_{R}$ are plotted in figure 8. From relations (18) and (20) they can be written in the simplified form:

$$
\begin{aligned}
{\left[\mathrm{Ar}_{2}^{+}\right] } & =n_{\mathrm{e}} /\left(1+\frac{\alpha_{\mathrm{D}}}{K \cdot n_{0}^{2}} n_{\mathrm{e}}\right) \\
\nu_{\mathrm{R}} & =K n_{0}^{2} /\left(1+\frac{K \cdot n_{0}^{2}}{\alpha_{\mathrm{D}} \cdot n_{\mathrm{e}}}\right) .
\end{aligned}
$$

Thus two physical limits appear:

- at low electron densities $\left(n_{\mathrm{e}} \ll 10^{13} \mathrm{~cm}^{-3}\right)$ [ $\left.\mathrm{Ar}_{2}^{+}\right]$is nearly equal to $n_{\mathrm{e}}$ and $\nu_{\mathrm{R}}$ is proportional to $n_{\mathrm{e}}$.

- at high electron densities $\left(n_{\mathrm{e}} \geqslant 10^{13} \mathrm{~cm}^{-3}\right)$ $\nu_{\mathrm{R}}$ and $\left[\mathrm{Ar}_{2}^{+}\right]$are independent of $n_{\mathrm{e}}$. Moreover [ $\left.\mathrm{Ar}_{2}^{+}\right]$is proportional to $n_{0}^{2}$.

Under our experimental conditions (10 Torr $\leqslant$ $p \leqslant 200$ Torr), the electron density ranges from about $10^{13} \mathrm{~cm}^{-3}$ at the end of the discharge to a fow $10^{14} \mathrm{~cm}^{-3}$ at the excitation gap (cf. Fig. 4). Hence $\nu_{R}$ and $\left[\mathrm{Ar}_{2}^{+}\right]$have only a weak dependence on $n_{\mathrm{e}}$, and thus are almost constant along the discharge (except at the end of the plasma column), in spite of the electron density gradient. Such calculations lead to about $10^{12} \mathrm{~cm}^{-3} \mathrm{Ar}_{2}^{+}$densities, which confirms the dominant role of $\mathrm{Ar}_{2}^{+}$in the discharge.

\section{Discussion and conclusion.}

5.1 DisCusSION. - As it was previously pointed out, $\theta_{\text {ion }}\left(=\mathrm{eV}^{+} \nu_{\text {loss }}^{\text {eff }}\right)$ is almost independent of the electron density under our experimental conditions. Thus we can unambiguously plot the estimated values of $\theta_{\mathrm{el}}, \theta_{\mathrm{ion}}$ and the experimental values of $\theta$ versus the pressure (cf. Fig. 9).

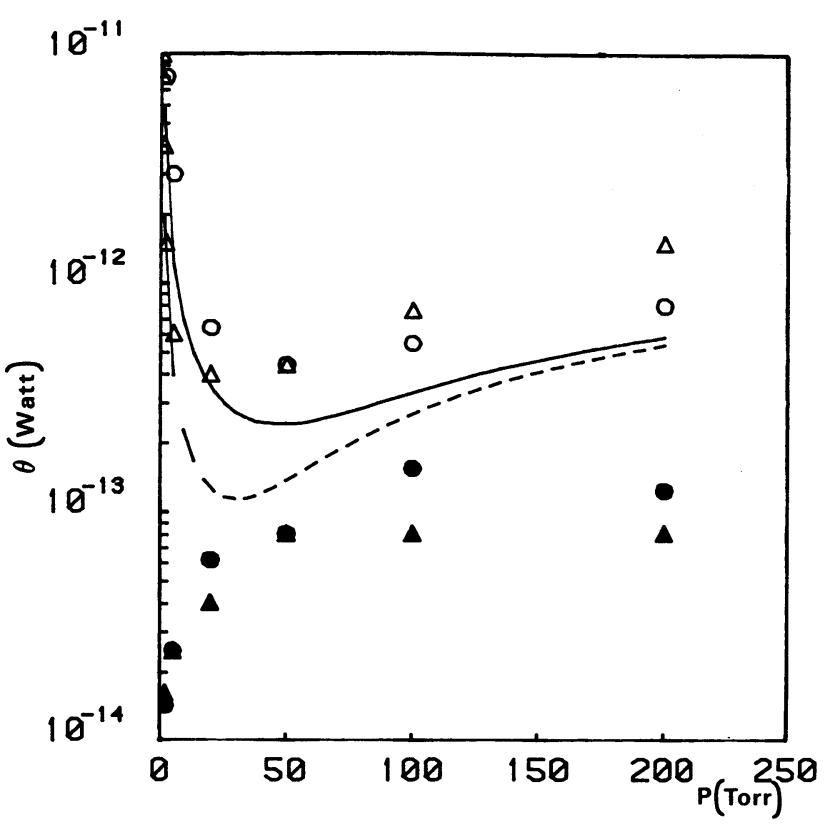

Fig. 9. - Estimated ănd experimental values of $\theta$ versus the pressure in several tubes assuming $T_{\mathrm{e}}=10^{4} \mathrm{~K}$. Experimental values of $\theta:(0), a=1 \mathrm{~mm} ;(\Delta), a=1.75 \mathrm{~mm}$; estimated $\theta_{\mathrm{el}}:(\bullet), a=1 \mathrm{~mm} ;(\Delta), a=1.75 \mathrm{~mm}$; estimated $\theta_{\text {ion }}:(-), a=1 \mathrm{~mm} ;(--), a=1.75 \mathrm{~mm}$.

$\theta_{\text {ion }}$ is plotted assuming $T_{0}$ increases linearly with $p$ from $1000 \mathrm{~K}$ at 10 Torr to $2000 \mathrm{~K}$ at 200 Torr. Anyway $T_{0}$ has not been measured and has been estimated from low pressure results and from higher pressure spectroscopic measurements [15]. The same neutral temperature has been used whatever the tube diameter; nevertheless we can expect $T_{0}$ to be lower in the largest tube, so that the dashed curve ought to be underestimated for medium pressures.

Firstly we note that $\theta_{\mathrm{el}}$ is small with regards to $\theta$, whatever the pressure.

Next we check that $\theta_{\text {ion }}$ is smaller than $\theta$ and moreover is about half of $\theta$.

We have previously pointed out that $\theta_{\text {ion }}$ is independent of the electron density, which is to link to the experimental result of independence of $\theta$ on $n_{\mathrm{e}}$ (i.e., on $Z$ ). Thus this independence is due to the $\mathrm{Ar}_{2}^{+}$kinetic in the discharge.

Moreover $\theta_{\text {ion }}$ and $\theta$ have similar variations with the pressure. Particularly $\theta_{\text {ion }}$ and $\theta$ exhibit their minimum at the same pressure, i.e. about 20 Torr in the $3.5 \mathrm{~mm}$ diameter tube and about 50 Torr in the $2 \mathrm{~mm}$ tube.

Thus the estimated $\theta_{\text {ion }}$ describes the behaviour of $\theta$ with both the electron density and the pressure quite well.

5.2 ConClusion. - Argon surface wave discharges have been characterized at medium pressure in 
capillary tubes. It has been experimentally pointed out that the mean power needed to maintain an electron in the discharge, $\theta$, is independent of the electron density and exhibits a minimum with the pressure.

A simple model of the energy balance, estimating the electron loss frequency, leads to approximate values of $\theta$. It points out that the main electron loss process is ambipolar diffusion up to $10 \mathrm{Torr}$ and dissociative recombination of $\mathrm{Ar}_{2}^{+}$above 100 Torr.
Furthermore the estimated values of $\theta$ have almost the same behaviour as the experimental values with both the electron density and the pressure.

The smaller the tube, the higher is the pressure of the minimum of $\theta$. This particular pressure is of some interest since the power needed to maintain a given number of electron is then minimized. In particular, discharges for chemical analysis by spectroscopical emission could operate at such pressures, high enough to excite the sample.

\section{References}

[1] Moisan, Beaudry, C., Leprince, P., Plasma Sci. PS3 (1975) 55.

[2] Marec, J., Bloyet, E., Chaker, M., Leprince, P. and NGHIEM, P., Elec. Breakdown and Discharges in Gases (Plenum Pub. Corp.) B, 1983, p. 347.

[3] Chaker, M., Nghiem, P., Bloyet, E., Leprince, P. and Marec, J., J. Physique Lett. 43 (1982) 71.

[4] Boisse-Laporte, C., Granier, A., Dervisevic, E., Leprince, P. and Marec, J., J. Phys. D 20 (1987) 197.

[5] Ferreira, C., J. Phys. D 14 (1981) 1811.

[6] Ferreira, C., J. Phys. D 16 (1983) 1673.

[7] Boisse-Laporte, C., Granier, A., Bloyet, E., Leprince, P. and Marec, J., J. Appl. Phys. 61 (1987) 1740.
[8] Granier, A., Boisse-Laporte, C., Leprince, P., Marec, J. and Nghiem, J. Phys. D 20 (1987) 204.

[9] Nghiem, P., Chaker, M., Bloyet, E., Leprince, P. and Marec, J., J. Appl. Phys. 53 (1982) 2920.

[10] Chen, C. J., J. Chem. Phys. 50 (1969) 1560.

[11] O'Malley, T. F., Cunningham, A. J. and Hobson, R. M., J. Phys. B 5 (1972) 2126.

[12] Fitzwilson, R. L. and Chanin, L. M., J. Appl. Phys. 44 (1973) 5337.

[13] Zander, A. T. and Hieftue, G. M., Appl. Spectrosc. 35 (1981) 357.

[14] Goode, S. R. and Baughman, K. W., Appl. Spectrosc. 38 (1984) 755.

[15] Moussounda, P. S., RAnson, P. and Mermet, J. MM., Spectrochim. Acta 40 B (1985) 641. 